\title{
Resíduos de Sisal como Reforço em Compósitos de Polipropileno Virgem e Reciclado
}

\author{
Francisco Rosário \\ Departamento de Engenharia de Materiais, UFSCar \\ Tecnologia em Polímeros, FATEC - Zona Leste, São Paulo/SP \\ Wagner M. Pachekoski \\ Centro Integrado de Manufatura e Tecnologia, Senai, Salvador/BA
}

\author{
Ana P. J. Silveira \\ Departamento de Farmácia, Unifenas \\ Sérgio F. dos Santos, Holmer S. Júnior \\ Grupo de Construções e Ambiência, USP
}

\author{
Suzan Aline Casarin \\ Departamento de Engenharia de Materiais, UFSCar
}

\begin{abstract}
Resumo: Foram estudadas as propriedades térmicas e mecânicas de compósitos de polipropileno, virgem e reciclado, reforçados com $30 \%$ em massa de fibras residuais de sisal, assim como o perfil de processamento e a morfologia da matriz polimérica. Para tanto, foram determinadas a resistência à tração, o módulo de Young, alongamento na ruptura, e energia de impacto. As amostras também foram caracterizadas por MEV, DMTA e TG. Para ambos os compósitos de polipropileno, virgem e reciclado, com a adição das fibras, o alongamento na ruptura mostrou uma queda significativa, enquanto que a resistência à tração não sofreu grandes variações. Houve um aumento significativo nos valores de tração na ruptura e de energia de impacto com a adição das fibras de sisal na matriz de polipropileno. As análises térmicas mostraram ligações secundárias, como as ligações polares, entre as fibras e a matriz, concordando com o comportamento mecânico dos compósitos. Constatou-se que a temperatura de transição vítrea não variou após a adição da fibra.
\end{abstract}

Palavras-chave: Sub-produto do sisal, extrusão, compatibilidade, propriedades mecânicas, propriedades térmicas.

\section{Virgin and Recycled Polypropylene Composites Reinforced with Sisal By-Product}

Abstract: The mechanical and thermal properties of virgin and recycled polypropylene composites reinforced with $30 \%$ by mass of residual sisal fibers were studied, in addition to an analysis of the extrusion process and morphology of the polymeric matrix. Tensile strength, Young's modulus, elongation at break, and impact energy were determined. The samples were also characterized by SEM, DMTA and TG analyses. Elongation at break of the composites presented a significant decrease, while the tensile strength was not affected significantly by addition of sisal fibers. A significant increase was observed in the tension of rupture and in the impact energy of the composite reinforced with sisal fiber. The thermal analyses indicated secondary interactions, such as polar interactions, between the fibers and the matrix, consistent with the mechanical behavior of the composites. The glass transition temperature has not changed after fiber addition.

Keywords: Sisal by-product, extrusion, compatibility, mechanical properties, thermal properties.

\section{Introdução}

O polietileno de baixa e alta densidade e polipropileno representam $45 \%$ da produção de plástico no mundo ${ }^{[1]}$. Particularmente, o polipropileno (PP) virgem é amplamente explorado, mas o aumento do custo das resinas plásticas, pressionado pelas constantes flutuações do preço do petróleo no mercado internacional, tem estimulado as pesquisas em reciclagem deste polímero. Normalmente, o preço do plástico reciclado é $40 \%$ mais baixo do que o da resina virgem ${ }^{[2]}$. Fernandes e Domingues analisaram-se as propriedades mecânicas de misturas de PP virgem e reciclado, em três porcentagens diferentes, por meio de ensaios de tração axial e de impacto Charpy. Esses pesquisadores concluiram que porcentagens de reciclado acima de $30 \%$ causam deterioração das propriedades mecânicas do produto ${ }^{[2]}$.

No entanto, há vários trabalhos na literatura que avaliam o reforço de fibras celulósicas em diversos tipos de matrizes poliméricas com objetivo de melhorar o desempenho mecânico e/ou compensarem possíveis degradações de compósitos poliméricos ${ }^{[3-10]}$. As fibras vegetais são abundantes, não-tóxicas, de baixo custo, de baixa densidade, não são abrasivas e, portanto, não desgastam os equipamentos, assim como provêm de fontes renováveis. Com isso, podem servir como um excelente agente de reforço para os polímeros, uma vez que suas propriedades mecânicas são comparáveis às de outros reforços comumente empregados. 
Dentre as fibras celulósicas mais utilizadas para reforçar matrizes poliméricas está a fibra de sisal. O Brasil é um grande produtor de fibras de sisal, toda ela concentrada na região Nordeste ${ }^{[11-12]}$. A produção nacional de fibras de sisal já alcançou 135 mil toneladas, destas, 113 mil foram exportadas, sendo $26 \%$ de fibras e $74 \%$ de manufaturados ${ }^{[9]}$.

A combinação de compósitos poliméricos com fibras de sisal se destaca por apresentar bom desempenho mecânico quanto à alta resistência ao impacto, assim como boas propriedades de resistência à tração axial e à flexão. Isto é atribuído ao fato da fibra de sisal apresentar um dos maiores valores de módulo de elasticidade e de resistência mecânica entre as fibras naturais ${ }^{[9,13-15]}$.

No presente trabalho estudaram-se os efeitos da incorporação das fibras de sisal provenientes da rasadeira (pedaços de fibras da fábrica de cordas), em uma matriz polimérica de polipropileno (PP) virgem e reciclado, nas propriedades mecânicas e térmicas do compósito.

\section{Revisão Bibliográfica}

\section{Polipropileno}

O polipropileno (PP) é um polímero termoplástico da classe das poliolefinas, essencialmente linear e altamente cristalino. Graças à excelente combinação de propriedades térmicas e mecânicas o PP é amplamente explorado numa variedade de aplicações que, combinada com condições favoráveis econômicas, encorajou uma rápida expansão no uso deste material. O PP pode ser modificado para uma variedade de aplicações, por meio de copolimerização, orientação e outras técnicas. As características físicas do polímero podem ser variadas para conseguir uma larga faixa de propriedades térmicas e mecânicas. A facilidade no processamento desse polímero permite o seu uso econômico na maioria das técnicas de fabricação comerciais ${ }^{[16-18]}$.

O polipropileno tem ponto de fusão superior ao da maioria das poliolefinas, em razão da maior rigidez estrutural ocasionada pela rotação do grupo lateral metil. Para o PP isotático, o ponto de fusão pode alcançar o valor máximo de $176^{\circ} \mathrm{C}$, enquanto para o $\mathrm{PP}$ sindiotático tal limite é de $135^{\circ} \mathrm{C}$. O ponto de fusão, em ambos os casos, é dependente da porcentagem de fase cristalina no polímero. Como o PP atático não cristaliza, não possui ponto de fusão e apresenta somente temperatura de transição vítrea.

Valores encontrados para a temperatura de transição vítrea variam muito, por causa do uso de diferentes técnicas de medidas e da influência da taticidade e da massa molar. Para o PP isotático, os valores de $\mathrm{Tg}$ vão de -13 a $0{ }^{\circ} \mathrm{C}$ e para a forma atática de -18 a $5{ }^{\circ} \mathrm{C}$. $\mathrm{O} \mathrm{PP}$, assim como as poliolefinas, é considerado biologicamente resistente a microorganismos. Além de não sofrer ataque biológico, o polipropileno não causa reações fisiológicas, podendo ser utilizado em fins terapêuticos, como por exemplo, em suturas. Graças a sua inércia química, é comum o uso de PP em embalagens de alimentos sensíveis a umidade e gordura.

O PP é altamente resistente a substâncias químicas. É, porém atacado por agentes oxidantes como ácido sulfúrico concentrado e ácido nítrico fumegante. Ele é solubilizado em solventes alifáticos de alto ponto de ebulição e hidrocarbonetos aromáticos em altas temperaturas ${ }^{[16-18]}$.

As propriedades mecânicas do polipropileno podem ser consideradas amplas devido à sua cristalinidade. Com ponto de fusão relativamente alto, a fase cristalina mantém resistência mecânica a altas temperaturas. À baixas temperaturas, o PP é limitado pela sua fragilização na temperatura de transição vítrea ( $\mathrm{Tg})$, em que as regiões amorfas se tornam vítreas. Para evitar tal fragilidade, o PP é normalmente copolimerizado com o etileno.

O polipropileno é de grande uso industrial e pode ser conformado segundo as principais técnicas de processamento ${ }^{[16-18]}$ :

- Moldagem por injeção: peças de PP moldadas por injeção são principalmente utilizadas na indústria automotiva. $\mathrm{O}$ polipropileno é amplamente utilizado também para a fabricação de vasilhames fechados e, graças à possibilidade de ser esterilizado, é também de grande uso médico.

- Filmes: filmes de PP são usados na indústria de embalagens de vários produtos e como componentes em sacolas.

- Termoformagem e Extrusão: as principais utilizações do PP termoformado são no ramo de vasilhames, como copos de iogurte e pote de margarina, por exemplo. Na forma extrudada, a principal utilização do PP é na fabricação de tubos e copos descartáveis transparentes ${ }^{[16]}$.

- Moldagem por sopro: garrafas de PP orientado possuem alta transparência e boa propriedade de barreira, sendo usadas em embalagens de detergentes, xampus e outros produtos.

\section{Sisal}

Sisal é uma planta de folhas pontiagudas e lanceoladas, muito grandes, dispostas na forma de roseta. O nome "sisal" foi emprestado de uma cidade portuária em Yucatan, Maya, México, e significa "água fria. As folhas do sisal têm de 8 a $10 \mathrm{~cm}$ de largura e de 150 a $250 \mathrm{~cm}$ de comprimento. De acordo com Tolêdo Filho et al., são compostas de mucilagem (15\%), fibras (3\%), bucha (1\%) e suco $(81 \%)$, e são usadas para os mais diversos fins ${ }^{[19]}$. As folhas são compostas de microfibras e contêm uma média de 1.100 fibras por folha, fibras resistentes à tração, duráveis e de comprimento individual variando de 1,5 a 4,0 mm e 10 a $30 \mu \mathrm{m}$ de largura, achandose distribuídas em toda a folha. Normalmente, com as fibras do sisal são tradicionalmente usadas para confeccionar fios, barbantes, cordas marítimas, sacarias, tapetes, estofamentos e artesanatos.

O processo de beneficiamento das fibras de sisal compreende-se as fases de desfibramento, lavagem, secagem, batimento, prensagem e enfardamento ${ }^{[1-12]}$. A fibra beneficiada de sisal é classificada, quanto à classe em longa (comprimento acima de $0,90 \mathrm{~m}$ ), média (comprimento entre 0,71 e $0,90 \mathrm{~m}$ ) e curta (comprimento entre 0,60 e $0,70 \mathrm{~m}$ ), e quanto ao tipo em Tipo superior, Tipo 1, Tipo 2 e Tipo 3, abaixo discriminados:

- Tipo superior: Material constituído de fibras lavadas, secas e bem batidas ou escovadas, de coloração creme-clara, em ótimo estado de maturação, com maciez, brilho e resistência bem acentuados, umidade máxima de $13,5 \%$, bem soltas e desembaraçadas, isentas de impurezas, de substâncias pécticas, de entrançamentos e nós, fragmentos de folhas e cascas, e de quaisquer outros defeitos.

- Tipo 1: Constituído de fibras secas e bem batidas ou escovadas, de coloração creme-clara ou amarelada, em ótimo estado de maturação, com maciez, brilho e resistência normais, manchas com pequena variação em relação à cor, umidade máxima de $13,5 \%$, soltas e desembaraçadas, isentas de impurezas, substâncias pécticas, entrançamentos e nós, fragmento de folhas e cascas, e de quaisquer outros defeitos.

- Tipo 2: Constituído de fibras secas e bem batidas ou escovadas, de coloração amarelada ou pardacenta, com pequenas extensões esverdeadas, em bom estado de maturação, com brilho e resistência normais, ligeiramente ásperas, umidade máxima de $13,5 \%$, soltas e desembaraçadas, isentas de impurezas, entrançamentos, nós e cascas.

- Tipo 3: Constituído de fibras secas e bem batidas ou escovadas, de coloração amarelada, com parte de tonalidade esverdeada, pardacenta ou avermelhada, em bom estado de maturação, com 
brilho e resistência normais, ásperas, manchas com variação bem acentuadas em relação à cor, umidade máxima de 13,5\%, soltas e desembaraçadas, isentas de impurezas, entrançamentos, nós e cascas.

\section{Compósitos}

Os compósitos poliméricos contendo fibras naturais têm recebido grande atenção por parte dos pesquisadores e das indústrias aeroespacial e automotiva, além de serem usados em aplicações militares e construção civil entre outras aplicações ${ }^{[3,8,20,21]}$. Fibras vegetais como juta, sisal, coco, kenaf e abacaxi têm sido utilizadas como reforço em matrizes poliméricas. Dentre essas fibras, a de sisal é particularmente interessante, já que seus compósitos possuem elevada resistência ao impacto, além de moderada resistência à tração axial e à flexão, se comparados a compósitos reforçados por outras fibras vegetais ${ }^{[9,22,23]}$. Além da grande importância sócioeconômica, ligada à cultura do sisal no Brasil, existem outros fatores que motivam seu estudo como reforço para polímeros, resultando em produtos ecologicamente corretos, nos quais é feita a substituição de fibras sintéticas pelas fibras naturais ${ }^{[12]}$.

Dentre os vários polímeros estão sendo usados como matrizes em compósitos reforçados com fibras de sisal, os mais comuns são os termofixos, tais como poliésteres, epóxi e fenólicos, e os termoplásticos, como o polietileno e o polipropileno. A incorporação de fibras de sisal a plásticos tem sido reportada por vários pesquisadores, que investigaram a viabilidade do desenvolvimento de compósitos poliméricos de baixo custo reforçados por fibras de sisal por meio da avaliação das propriedades mecânicas, reológicas, elétricas e viscoelásticas desses compósitos ${ }^{[4-6,9,24-27]}$.

Alguns pesquisadores ${ }^{[6,22,23,28-32]}$ avaliaram as propriedades mecânica de impacto de compósitos poliméricos. Em especial, o elevado trabalho de fratura obtido para os compósitos reforçados por sisal, apesar de estas fibras apresentarem problemas de adesividade, porquê as fibras celulósicas são hidrofílicas não aderem muito bem em matrizes poliméricas hidrofóbicas, e baixos valores de módulo de Young ${ }^{[29]}$. Além disso, há trabalho que indicam que a previsão das propriedades de impacto de compósitos reforçados por fibras vegetais não será válida se a contribuição da estrutura helicoidal microfibrilar da fibras vegetal não for considerada ${ }^{[26]}$.

É fato geralmente aceito que a tenacidade de compósitos reforçados por fibras depende primordialmente do comportamento tensão-deformação da fibra. Fibras resistentes com alta deformação de ruptura elevam o trabalho de fratura dos compósitos. Bisanda e Ansell ${ }^{[27]}$ avaliaram as propriedades de compósitos epóxi/sisal e reportaram que a incorporação do sisal à matriz epóxi gera produtos rígidos e resistentes.

\section{Parte Experimental}

\section{Materiais}

As fibras de sisal, fornecidas pela Associação de Desenvolvimento Sustentável e Solidário da Região Sisaleira (Apaeb), sediada em Valente, BA, são fibras descartadas no beneficiamento, foram lavadas com água, secas em estufa e cortadas nas dimensões desejadas. Com base no tamanho dos péletes, que são picotados no processo de extrusão, se a fibra for maior que o pélete, uma parte dela será retirada da matriz polimérica. As que não forem arrancadas serão maiores que o pélete e isto diminui o empacotamento e dificulta a alimentação no processo e de injeção. As fibras foram utilizadas como reforço em compósitos com duas matrizes de polipropileno (PP) de origem diferentes, PP virgem homopolímero H 103 da Braskem e PP reciclado tipo canelinha fornecido pela Indústria de Plástico Lombarda, situada na cidade de São Paulo, SP. As Figuras 1 e 2 mostram, respectivamente, a estrutura química do PP e a fibra de sisal.

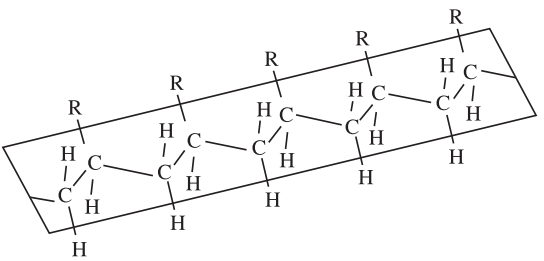

(a)

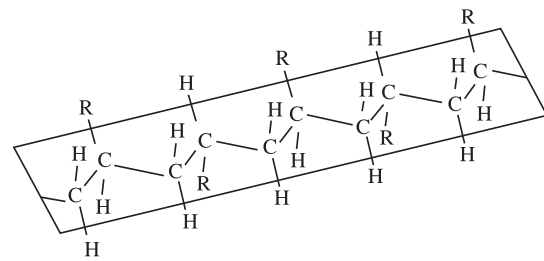

(b)

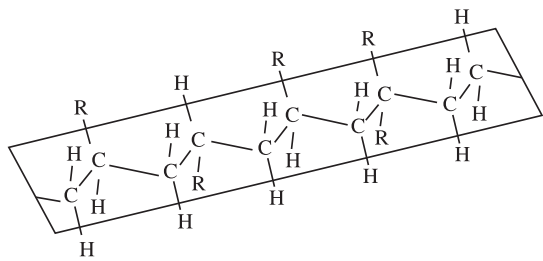

(c)

Figura 1. Estrutura química do PP: a) isotático; b) sindiotático; e c) atático.

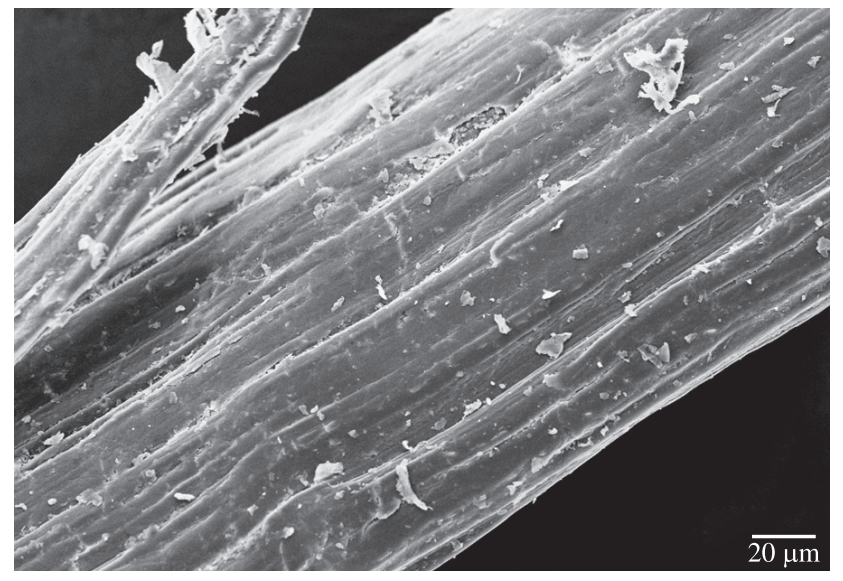

(a)

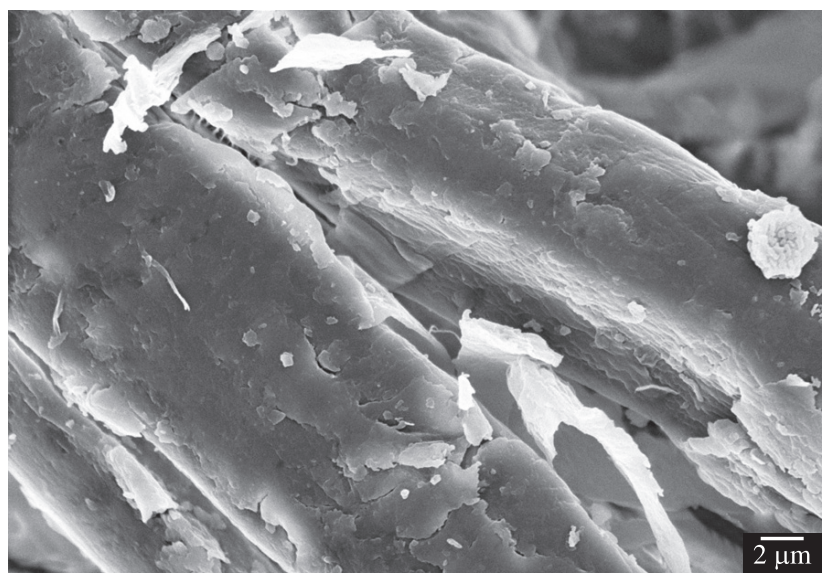

(b)

Figura 2. a) Micrografia da superfície e b) detalhe indicando a rugosidade da superfície da fibra de sisal. As micrografias foram realizadas com o detector no modo elétrons secundários. 


\section{Métodos}

As fibras de sisal foram lavadas com água por 1 hora, a $80{ }^{\circ} \mathrm{C}$ e secas em estufa a $60{ }^{\circ} \mathrm{C}$ por 6 horas e picotadas em um picotador mecânico, reduzindo o comprimento para aproximadamente de 2 a $4 \mathrm{~mm}$.

O método utilizado neste trabalho consistiu em se fazer a mistura dos materiais (fibra de sisal e PP) antes do processamento. Trata-se de mistura manual em recipiente (saco plástico), seguindo a proporção em massa de cada material na mistura (70\% em massa de PP e $30 \%$ em massa de fibra). Essa mistura heterogênea é colocada no alimentador. Para qualquer velocidade de dosagem na extrusora, sempre é mantida a porcentagem em massa dos elementos da mistura e esta é alimentada na extrusora de dupla rosca com perfil para mistura e incorporação das fibras à matriz polimérica. Após a extrusão do compósito com $30 \%$ em massa de fibra de sisal à temperatura aproximada de $200{ }^{\circ} \mathrm{C}$, foi feita a secagem por 48 horas em estufa com circulação de ar a $60{ }^{\circ} \mathrm{C}$. Por último, realizou-se o processamento de injeção em injetora Arburg All Rounder modelo 270V 300 -120, dos corpos-de-prova para os ensaios de tração e impacto.

Os compósitos, com fibras curtas de sisal aleatoriamente distribuídas, foram caracterizados por meio de ensaio mecânico de resistência à tração, microscopia eletrônica de varredura (MEV), análise térmica dinâmico-mecânica (DMTA) e análise por termogravimetria (TG).

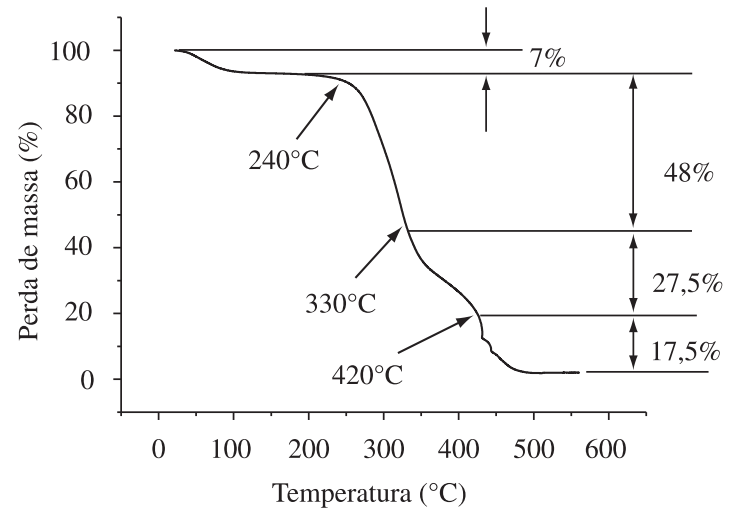

(a)

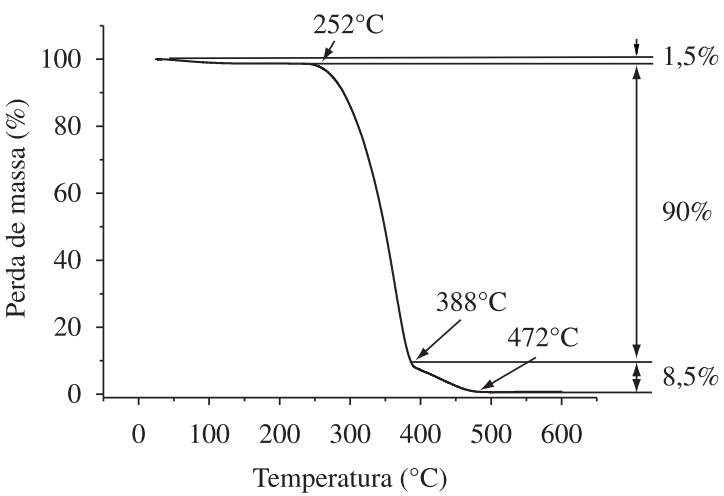

(c)

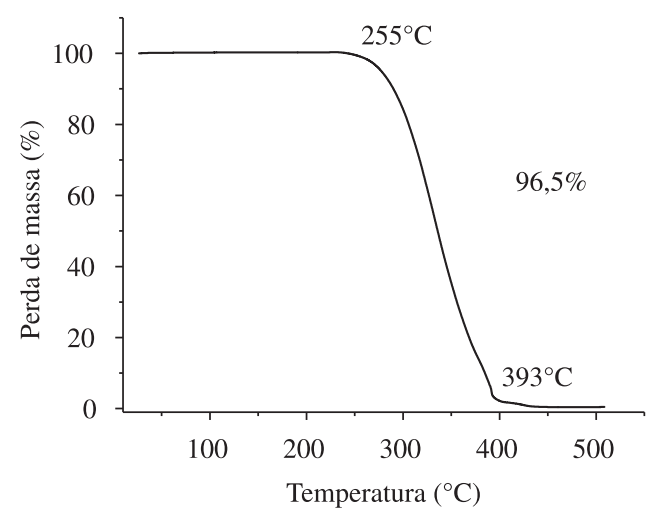

(b)

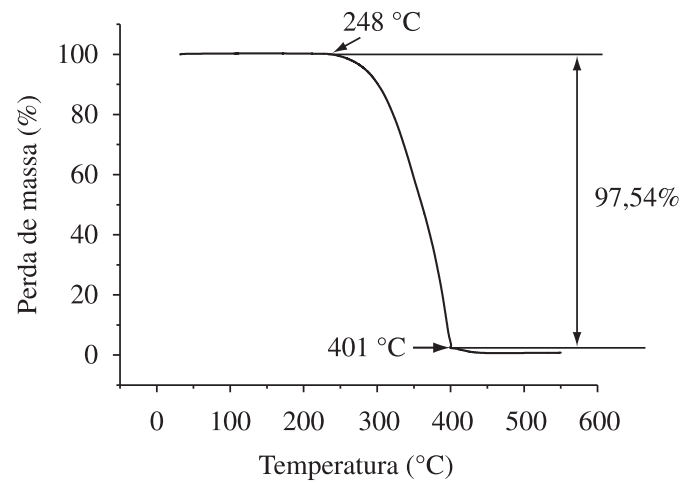

(d)

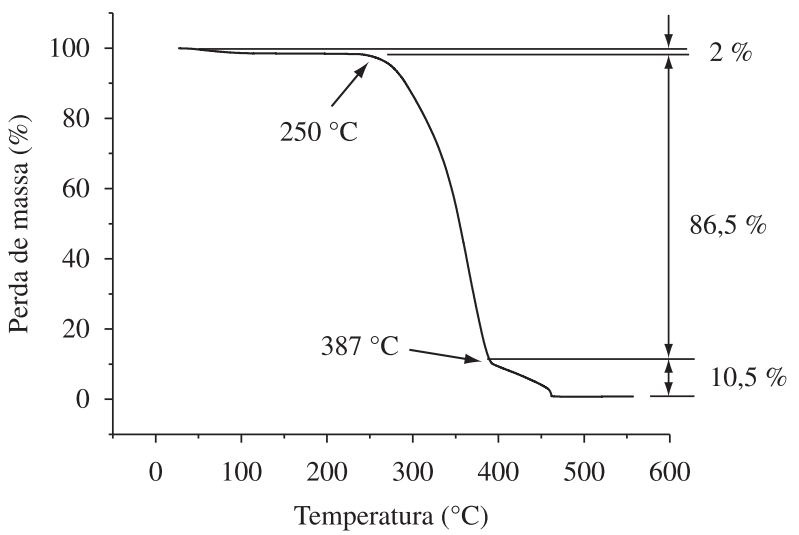

(e)

Figura 3. Análise termogravimétrica: a) fibra de sisal; b) PP virgem sem sisal; c) PP virgem com $30 \%$ de fibra sisal; d) PP reciclado sem sisal; e e) PP reciclado com sisal. 


\section{Análise termogravimétrica (TG)}

A TG foi realizada com o equipamento TGA 2950 da TA Instruments. As amostras foram aquecidas a partir da temperatura ambiente (aproximadamente $25^{\circ} \mathrm{C}$ ) até $600{ }^{\circ} \mathrm{C}$, a uma taxa de aquecimento de $20^{\circ} \mathrm{C} / \mathrm{min}$ em atmosfera inerte de nitrogênio $\left(\mathrm{N}_{2}\right)$. Os ensaios foram realizados em duplicata.

\section{Ensaios mecânicos}

Os ensaios mecânicos de tração e impacto foram realizados com os corpos-de-prova injetados. Os testes de tração foram realizados na máquina universal de ensaios Instron, modelo 5569, segundo a norma ASTM D-638 ${ }^{[33]}$, com a distância entre garras $1_{0}$ correspondente a $115 \mathrm{~mm}$, velocidade de ensaio de $50 \mathrm{~mm} / \mathrm{min} \mathrm{e}$ célula de carga de $50 \mathrm{kN}$.

Os corpos-de-prova, confeccionados segundo a norma, apresentam as seguintes dimensões: Comprimento: $165 \pm 6,5 \mathrm{~mm}$, comprimento útil igual $57,0 \pm 2,3 \mathrm{~mm}$; Largura da seção estreita $=13,0 \pm 0,5 \mathrm{~mm}$ e espessura igual a 3,28 $\pm 0,04 \mathrm{~mm}$. Foram realizados sete ensaios e o valor apresentado é uma média.

Os corpos-de-prova para os ensaios de impacto foram entalhados e testados na máquina de impacto Izod Ceast, modelo 545100 , modelo Resil, com martelo de $2 \mathrm{~J}$, temperatura controlada e segundo norma ASTM D-256 ${ }^{[34]}$. O valor de resistência ao impacto foi obtido pelo cálculo da média dos resultados de sete amostras avaliadas. Os corpos-de-prova, na forma de barras retangulares, segundo a norma, apresentam as seguintes dimensões: comprimento: $=61,9 \pm 2,3 \mathrm{~mm}$, largura igual a $12,70 \pm 0,15 \mathrm{~mm}$ e espessura de $3,25 \pm 0,03 \mathrm{~mm}$.

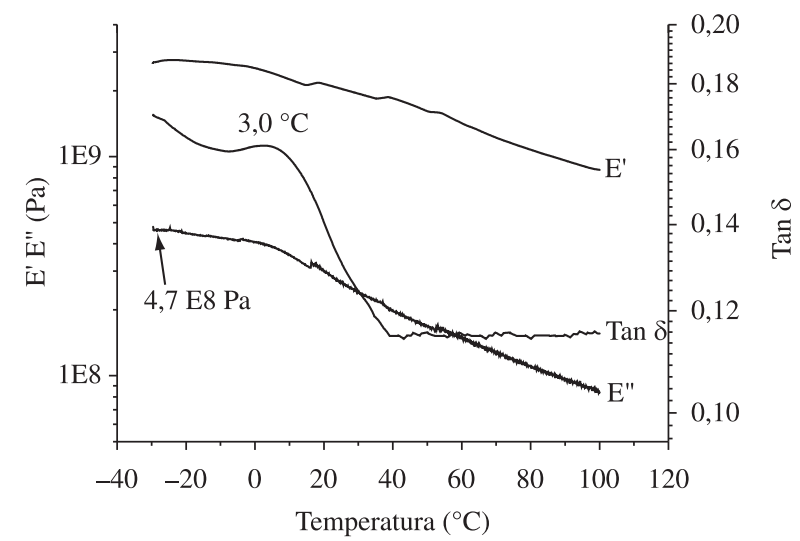

(a)

\section{Análise térmica}

O ensaio de análise térmica dinâmico-mecânica (DMTA) foi realizado no equipamento DMA 2980 Dynamic Mechanical Analyzer da TA Instruments do Departamento de Química do grupo de Físico Química Orgânica do Instituto de Química de São Carlos da USP, no modo flexão à três pontos, segundo a norma ASTM D 502313. A faixa de temperatura utilizada foi de 25 a $100{ }^{\circ} \mathrm{C}$, com frequência de $1 \mathrm{~Hz}$ e taxa de aquecimento de $2{ }^{\circ} \mathrm{C} / \mathrm{min}$. Foram realizados ensaios em duplicata. Com esse teste, foi possível avaliar o comportamento dinâmico-mecânico dos materiais em estudo (PP e compósitos) para posterior comparação entre os dados obtidos.

\section{Microscopia Eletrônica de Varredura (MEV)}

Para as análises de MEV, utilizou-se um aparelho Stereoscan 440 , com detector de elétrons secundários. Todas as amostras foram fraturadas criogenicamente e recobertas de ouro.

\section{Resultados e Discussão}

\section{Termogravimetria (TG)}

Este experimento foi realizado com os objetivos de observar a eficácia do tempo de secagem dos materiais puros (PP virgem, PP reciclado e fibra de sisal) e analisar as temperaturas de decomposição dos materiais puros e em compósitos, avaliando-se a perda de massa em função da temperatura. Na Figura 3 são encontrados exemplos das curvas obtidas para os diversos materiais analisados.

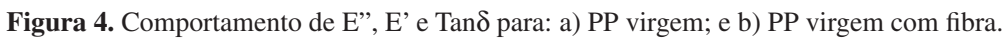

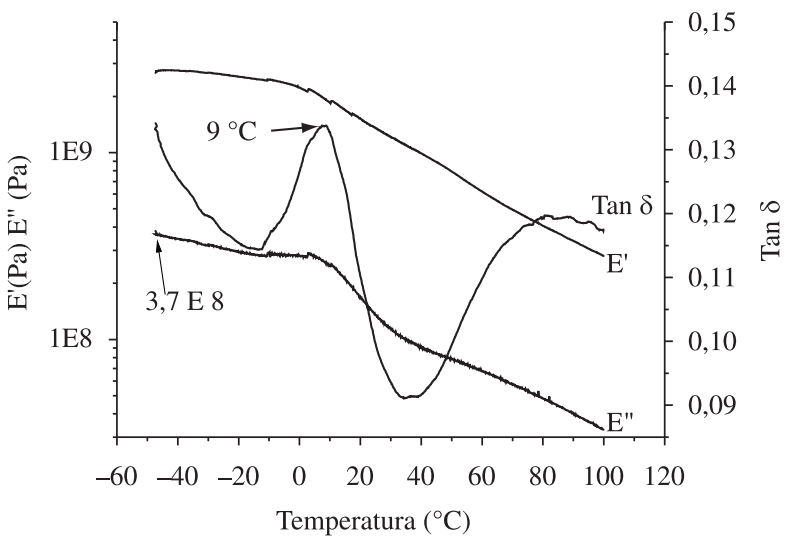

(a)

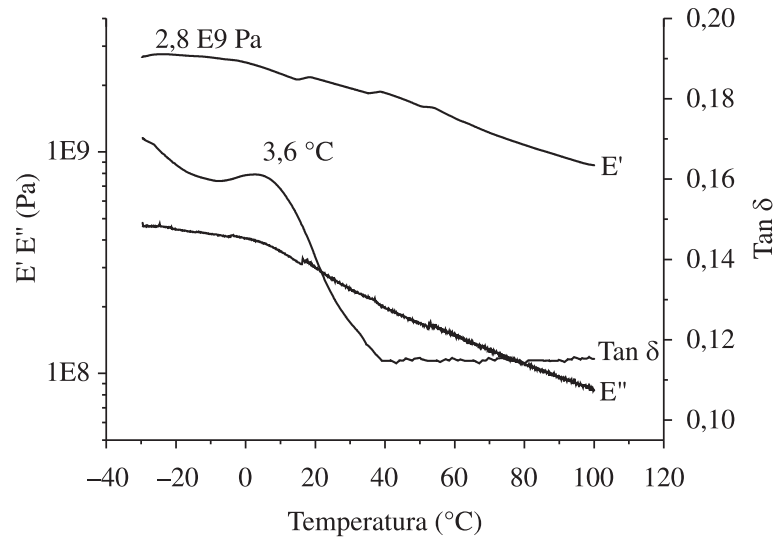

(b)

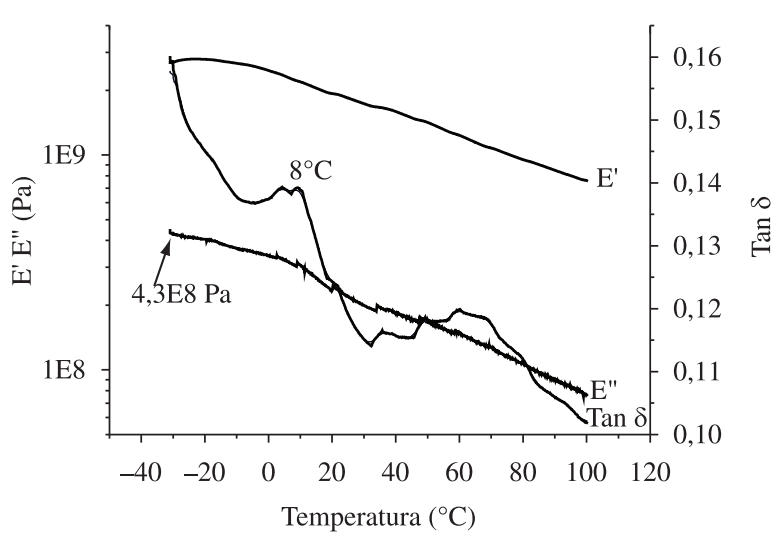

(b)

Figura 5. Comportamento de E", E' e Tan $\delta$ para: a) PP reciclado; e b) PP reciclado com fibra. 
$\mathrm{Na}$ análise das curvas de TG observou-se que a fibra de sisal possui umidade de $7 \%$ e o início de sua decomposição ocorre perto de $240{ }^{\circ} \mathrm{C}$ (Figura 3a). A matriz polimérica virgem tem a temperatura de decomposição inicial um pouco acima da fibra de sisal, aproximadamente $255^{\circ} \mathrm{C}$ (Figura 3b). O polipropileno reciclado, mostrado na Figura 3d, apresenta temperatura inicial de decomposição de aproximadamente $248^{\circ} \mathrm{C}$, que é menor que aquela do PP virgem. Isso se deve à degradação prévia sofrida nas várias fases de processamento em que o polímero foi submetido ao ser reciclado. As curvas das Figuras 3c e 3e mostram que os compósitos apresentam temperatura inicial de decomposição próximas à dos materiais puros com pouca influencia da fibra na resistência térmica a temperatura de degradação.

\section{Análise térmica dinâmico-mecânica (DMTA)}

Foram analisadas as curvas oriundas da componente da tensão resposta em fase com a deformação, E', denominada módulo de armazenamento. O módulo oriundo da tensão resposta $90^{\circ}$, fora de fase com a deformação, E”, é denominado módulo de perda. Assim, um material viscoelástico será caracterizado por dois valores de módulo, E' e E’. As Figuras 4 e 5 mostram o comportamento do PP virgem e reciclado e seus respectivos compósitos com sisal.

$\mathrm{Na}$ literatura, ainda não há um consenso sobre qual variável usar e de que forma determinar a temperatura de transição vítrea

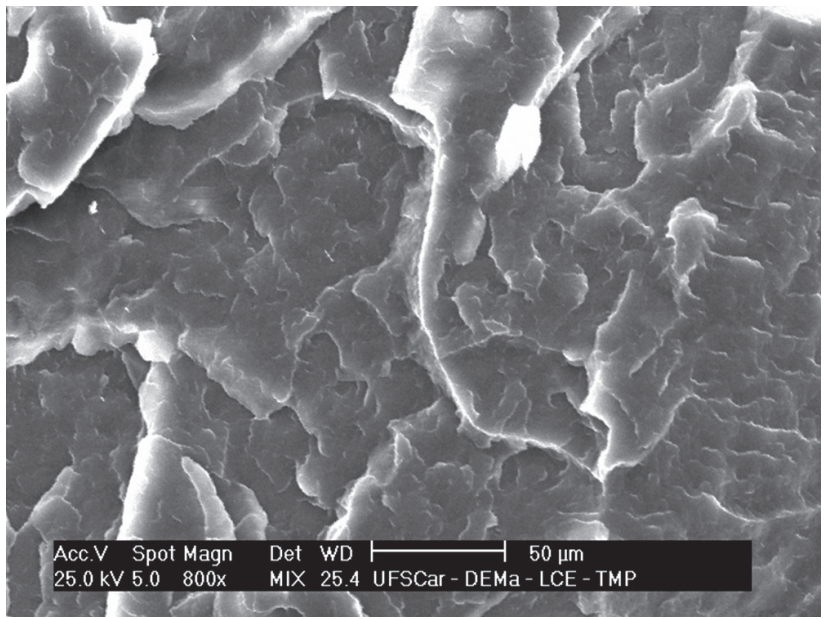

(a) nesta análise. Vários métodos são propostos: a temperatura do início da queda em E'; do início, ou do pico nas curvas de E" ou $\tan \delta$. Neste trabalho, foi usada a temperatura do pico da curva de $\tan \delta$, que é obtida pela relação entre os módulos de elasticidade (E'/E'). Essa escolha se explica pela constância do valor da temperatura de transição vítrea definido por esta variável.

Foi utilizado o método de flexão a três pontos. O comportamento térmico dinâmico-mecânico está mostrado nas Figuras 4 e 5, que apresentam os resultados de E', E" e tan $\delta$ em função da temperatura.

Por meio da análise das curvas $5 \mathrm{a}$ e $5 \mathrm{~b}$, concluiu-se que houve pequena ou quase nenhuma mudança nas relaxações $\delta(\mathrm{Tg})$, quando se compara o PP reciclado com o compósito formado por PP reciclado e sisal. O mesmo comportamento é evidenciado nas Figuras $4 \mathrm{a}$ e $4 \mathrm{~b}$, em que aparecem os materiais PP virgem e PP virgem com sisal. As curvas de DMTA dos polímeros puros e dos compósitos mostraram que não houve interferência nas propriedades térmica e mecânica com a incorporação das fibras. Evidenciou-se pouca interação entre a interface da fibra-matriz polimérica, em razão da incompatibilidade entre a matriz e a fibra.

\section{Microscopia eletrônica de varredura (MEV) dos compostos PP/fibra de sisal (70/30), PP puro e reciclado e microscopia óptica da fibra de sisal}

A utilização da microscopia eletrônica de varredura teve como objetivo avaliar a interface fibra de sisal e matriz, as superfícies

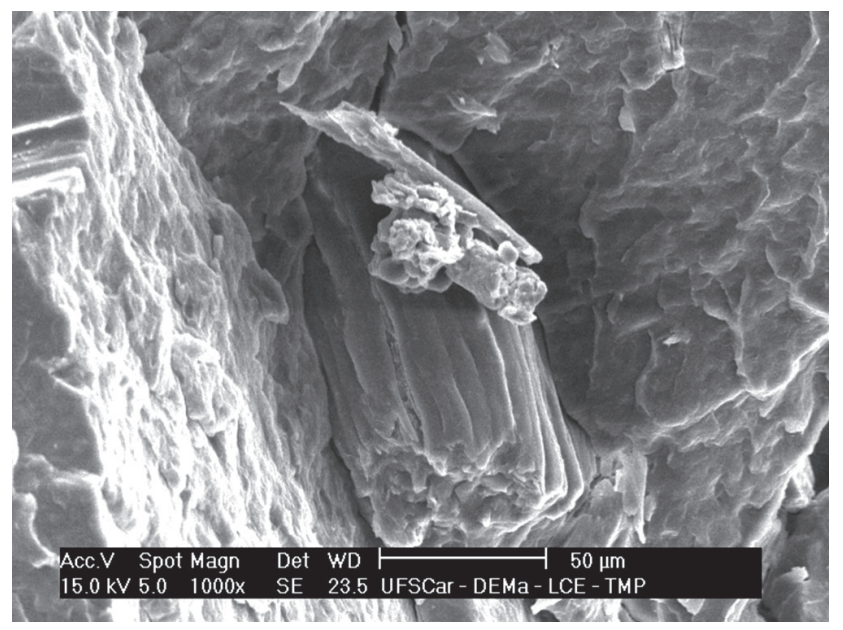

(b)

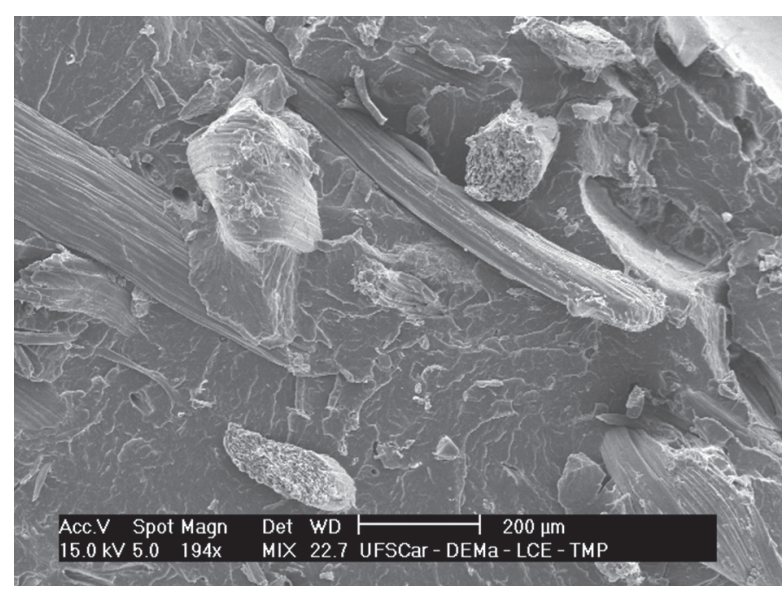

(c)

Figura 6. Microscopia Eletrônica de Varredura: a) matriz polímero virgem; b) compósito polímero virgem/sisal; e c) compósito polímero reciclado/sisal. 
Tabela 1. Resultados de ensaio mecânico.

\begin{tabular}{ccccc}
\hline Material & $\begin{array}{c}\text { Módulo de Young } \\
(\mathbf{G P a})\end{array}$ & $\begin{array}{c}\text { Tensão na ruptura } \\
(\mathbf{M P a})\end{array}$ & $\begin{array}{c}\text { Deformação } \\
(\mathbf{\%})\end{array}$ & $\begin{array}{c}\text { Impacto Izod } \\
\text { com entalhe } \\
(\mathbf{J} / \mathbf{m})\end{array}$ \\
\hline PP virgem & $1,78 \pm 0,09$ & $37,36 \pm 1,87$ & $33,69 \pm 1,69$ & $25,05 \pm 1,6$ \\
PP virgem + sisal & $3,03 \pm 0,15$ & $34,36 \pm 1,65$ & $2,81 \pm 0,17$ & $59,48 \pm 2,1$ \\
PP reciclado & $1,30 \pm 0,08$ & $22,78 \pm 1,14$ & $59,36 \pm 3,56$ & $26,25 \pm 4,5$ \\
PP reciclado + sisal & $2,87 \pm 0,14$ & $33,87 \pm 1,69$ & $3,51 \pm 0,21$ & $62,29 \pm 2,1$ \\
\hline
\end{tabular}

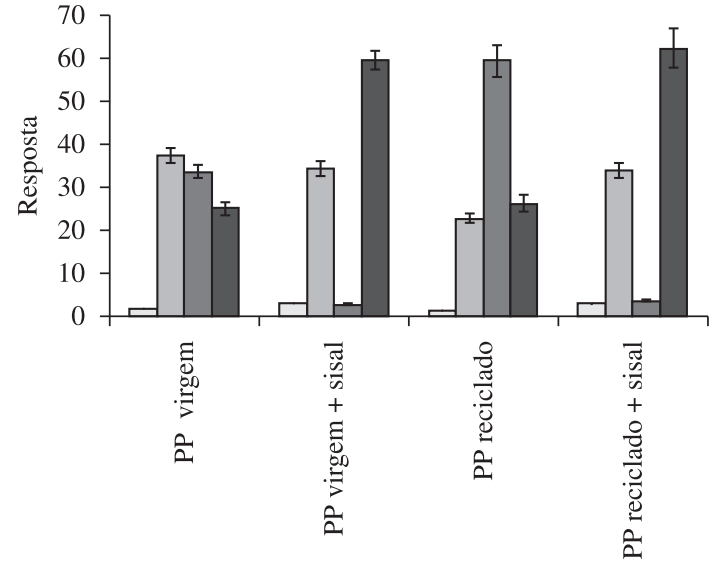

Tipo de amostra

\begin{tabular}{|ll}
\hline Módulo de young (Gpa) & $\square$ Tensão na ruptura $(\mathrm{Mpa})$ \\
$\square$ Deformação $(\%)$ & $\square$ Energia de impacto $(\mathrm{J} / \mathrm{m})$
\end{tabular}

Figura 7. Comparação entre os resultados do ensaio mecânico para os quatro tipos de amostras analisadas.

de fratura dos compósitos e seus aspectos morfológicos gerais. Foram obtidas micrografias dos compostos PP/fibra. A micrografia da Figura 6a mostra a matriz polimérica PP Virgem, enquanto nas Figuras 6 b e 6c são mostrados os compósitos de PP virgem/sisal e $\mathrm{PP}$ reciclado sisal respectivamente.

As Figuras 6b e 6c mostram os compósitos, com polímero virgem e reciclado respectivamente, evidenciando a interface entre ambos, a boa dispersão da fibra e a pouca adesão fibra/matriz. A pouca adesão conseguida entre a matriz e a fibra se deve à pouca compatibilidade entre a fibra celulósica, que tem energia superficial predominantemente polar e o polímero que tem energia apolar.

\section{Propriedades mecânicas}

\section{Resistência à Tração}

Foram realizados ensaios de tração para o polipropileno virgem, reciclado e seus compostos com fibra. Os resultados são apresentados na Tabela 1 e na Figura 7.

Com base nos resultados apresentados na Tabela 1 e na Figura 7 , nota-se que os sistemas de matriz de PP virgem contendo fibra apresentaram menor resistência à tração do que a matriz de polímero puro virgem. Tal fato pode ser atribuído à incompatibilidade entre o sisal e a matriz polimérica (baixa adesão sisal/matriz). No caso dos sistemas PP reciclado e PP reciclado/sisal, o comportamento foi contrário. Isso se justifica pela maior compatibilidade fibra de sisal com a matriz polimérica (PP) reciclada, por causa da distribuição de peso molecular (DPM) mais larga, com número maior de moléculas de baixa massa molar, o que contribui para o aumento da adesão matriz/fibra. Na análise das propriedades mecânicas dos sistemas com polímeros puros, nota-se que o material virgem apresentou módulo de Young ligeiramente superior. A resistência à tração na ruptura do polímero virgem também superou aquela do polímero puro reciclado. $\mathrm{O}$ alongamento na ruptura para o polímero reciclado foi superior provavelmente pela sua distribuição de peso molecular.

\section{Energia de Impacto}

A energia de impacto é um importante aspecto do comportamento mecânico de polímeros rígidos, pois as solicitações de impacto durante o uso de produtos são muito frequentes. Os resultados do ensaio de impacto Izod, com entalhe, para os diversos sistemas em estudo são mostrados na Tabela 1 e Figura 7.

A energia de impacto com entalhe teve aumentou o dobro no sistema com fibra de sisal. Este fato se explica pela orientação longitudinal da fibra na injeção do corpo-de-prova e perpendicular à propagação da trinca, induzida pelo entalhe, tornando-se mais difícil de romper o sistema PP/fibra.

Analisando a energia de impacto, percebe-se que o comportamento dos polímeros puros é similar e o mesmo ocorre entre os compostos polímero/sisal, independentemente de se tratar de polímero virgem ou reciclado.

\section{Conclusão}

$\mathrm{O}$ uso da matriz polimérica reciclada mostrou-se perfeitamente viável nas condições estudadas. Os compósitos com os resíduos de sisal apresentaram melhor desempenho em relação à matriz reciclada sem fibra, no que se refere à resistência à tração, módulo de Young e especialmente resistência ao impacto (aumento de 140\%). A boa dispersão e a mistura da fibra de sisal na matriz polimérica são atribuídas à utilização da dupla rosca. A análise termogravimétrica (TG) mostrou que a fibra de sisal não deve sofrer degradação ao ser processada com a matriz polimérica de polipropileno reciclada e virgem, pois sua temperatura de decomposição está bem acima da temperatura de processamento do compósito. A única propriedade mecânica que foi afetada acentuadamente de forma negativa, quando se adiciona a fibra como reforço, foi a deformação na ruptura. Este problema pode ser minimizado com um prévio tratamento na fibra de sisal para melhorar a sua adesão e ter uma maior compatibilidade com a matriz polimérica. E os resultados mostram a viabilidade do uso de matrizes recicladas de polipropileno com a fibra de resíduos de sisal.

\section{Agradecimentos}

Os autores agradecem ao Departamento de Engenharia de Materiais da Universidade Federal de São Carlos pelo suporte técnico e à FAPESP, à Finep e ao CNPq pelo apoio financeiro.

\section{Referências Bibliográficas}

1. Meran, C.; Ozturk, O. \& Mehmet, Y. - Mater. Des., 29, p.701 (2008).

2. Fernandes, B. L. E. \& Domingues, A. J. - Polímeros, 17, p.85 (2007).

3. Thomas, S. \& Pothan, L. A. - "Natural fibre reinforced polymer composites: macro to nanoscale", Old City Publishing (2009).

4. Suppakarn, N. \& Jarukumjorn, K. - Comp. Part B: Eng., 40, p.613 (2009). 
5. Sangthong, S.; Pongprayoon, T. \& Yanumet, N. - Comp. Part A: Appl. Sci. Manufact., 40, p.687 (2009).

6. Megiatto Jr, J. D.; Silva, C. G.; Ramires, E. C. \& Frollini, E. - Polym. Test., 28, p.793 (2009).

7. Kuruvilla, J., Siby, V.; Kalaprasada, G., Thomasa, S. L.; Prasannakumarib, P. K. \& Pavithranb, C. - Eur. Polym. J., 32, p.1243 (1996).

8. De Rodriguez, N. L. G.; Thielemans, W. \& Dufresne, A. - Cellulose, 13, p.261 (2006).

9. Martin, A. R.; Martins, M. A.; Mattoso, L. H. C. \& Silva, O. R. R. F. Polímeros, 19, p.40 (2009).

10. Mattoso, L. H. C.; Ferreira, F. C. \& Corvelo, A. A. S. - «Lignocellulosicplastics composites», UNESP/IPRONATI/ABPol, São Paulo (1998).

11. Embrapa Algodão - «Sistemas de Produção», 5, versão eletrônica (ISSN 1678-8710) (2006).

12. Santos, D’A. J. - «Produção e consumo», in: O sisal no Brasil, Andrade, W. (org.), cap.4, SINDIFIBRAS, Brasília, DF. (2006).

13. Bledzki, A. K. \& Gassan, J. - Prog. Polym. Sci., 24, p.221 (1999).

14. Couto, N. S.; Pereira N. C. \& Miranda L. F. - Proceedings of the Polymer Processing Society $24^{\text {th }}$ Annual Meeting PPS-24, Salerno Italy, June (2008).

15. Santana, R. M. C. \& Teixeira D. B. - Proceedings of the Polymer Processing Society $24^{\text {th }}$ Annual Meeting PPS-24, Salerno - Italy, June (2008).

16. PP Handbook, Hansen Publisers, New York (1996).

17. Maier, C. \& Calafut, T. - "Polypropylene the definitive user's guide and databook", Plastics Design Library, Norwich, NY, USA (1998).

18. Canevarolo, S. V. - "Ciência de Polímeros", 2. ed., Artliber, São Paulo (2007).
19. Tolêdo Filho, R. D.; Nascimento, J. W. B. \& Barreto, R. L. - in: Anais do XXI Congresso Brasileiro de Engenharia Agrícola, Santa Maria, RS (1992).

20. Martins, M. A.; Mattoso, L. H. C. - J. Appl. Polym. Sci., 91, p.670 (2004).

21. Georgopoulos, S. T. ; Tarantili, P. A. \& Avgerinos, E. A. - Polym. Degrad. Stab., 90, p.303 (2005).

22. Pavithran, C. et al. - J. Mater. Sci., 26, p.455 (1991).

23. Pavithran, C. et al. - Mater. Sci. Lett., 7, p.825 (1988).

24. Joseph, K. et al. - Reinf. Plast. Comp., 12, p.139 (1993).

25. Manikandan Nair, K. C. et al. - J. of Appl. Polym. Sci., 60, p.1483 (1996).

26. Paramasivan, V. \& Abdulkalam, A. P. J. - Fibre. Sci. Tech., 7, p.85 (1974).

27. Bisanda, E. T. N. \& Ansell, M. P. - Comp. Sci. Technol., 41, p.165 (1991).

28. Tai, C. M.; Li, R. K. Y. \& Ng, C. N. - Polym. Test., 19, p.143 (2000).

29. Antich, P.; Vázquez, A.; Mondragon, I. \& Bernal C. - Compos. Part A: Appl. Sci. Manufact., 37, p.139 (2006).

30. Doan, T-T-L.; Brodowsky, H. \& Mäder, E. - Compos. Sci. Technol., 67, p.2707 (2007).

31. Cui, Y.; Lee, S.; Noruziaan, B.; Cheung, M. \& Tao, J. - Composit. Part A: Appl. Sci. Manufact., 39, p.655 (2008).

32. Ashori, A. \& Nourbakhsh, A. - Biores. Technol., 101, p.2515 (2010).

33. ASTM. - "ASTM D638: Standard Test Method for Tensile Properties of Plastics", Philadelphia (2001).

34. ASTM - "ASTM D256: Standard Test Methods for Determining the Izod Pendulum Impact for Plastics", Philadelphia (2001).

Enviado: 23/11/09

Reenviado: $12 / 07 / 10$

Aceito: $27 / 08 / 10$

DOI: $10.1590 /$ S0104-14282011005000021 